SYMMETRICAL OSTEOMYELITIS FOLLOWED BY COMPLETE REGENERATION

George Blackburne, M.D., Newark, N. J.

Assistant Attending Surgeon, St. Michael's Hospital; Assistant Attending Gynecologist, Newark City Hospital

M. R., white girl, aged 12 years, was referred to me in December, 1914, with the following history: Five months

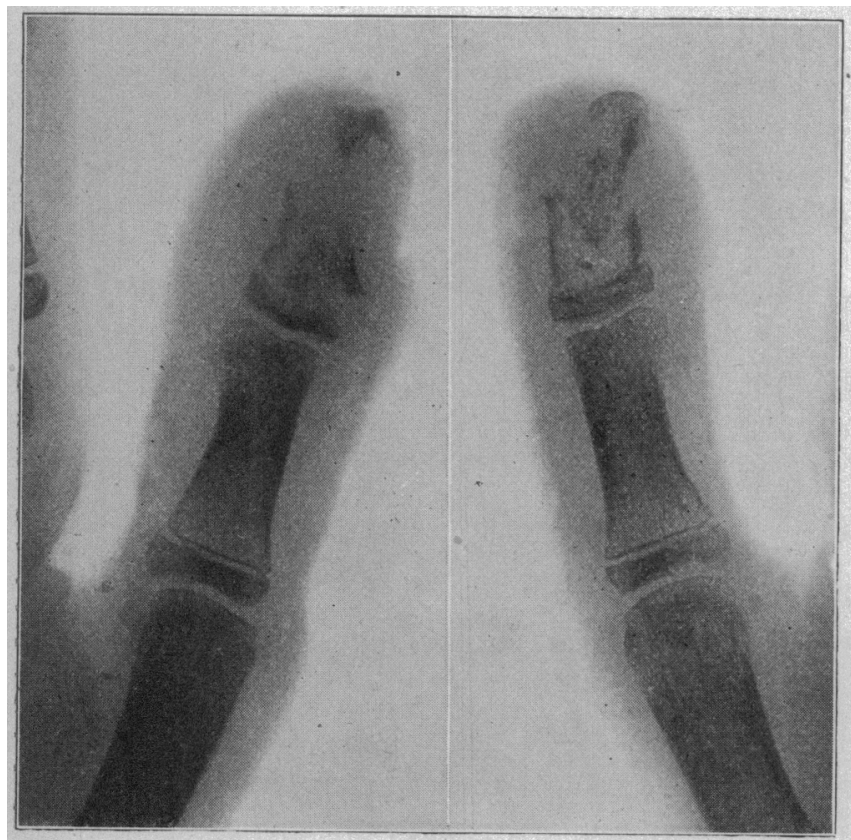

Fig. 1.-Condition of thumbs, Dec, 28, 1914.

before, the ends of both thumbs had become swollen and painful. A pustule appeared on the ball of each thumb. These pustules were incised by the patient's physician, evacuated

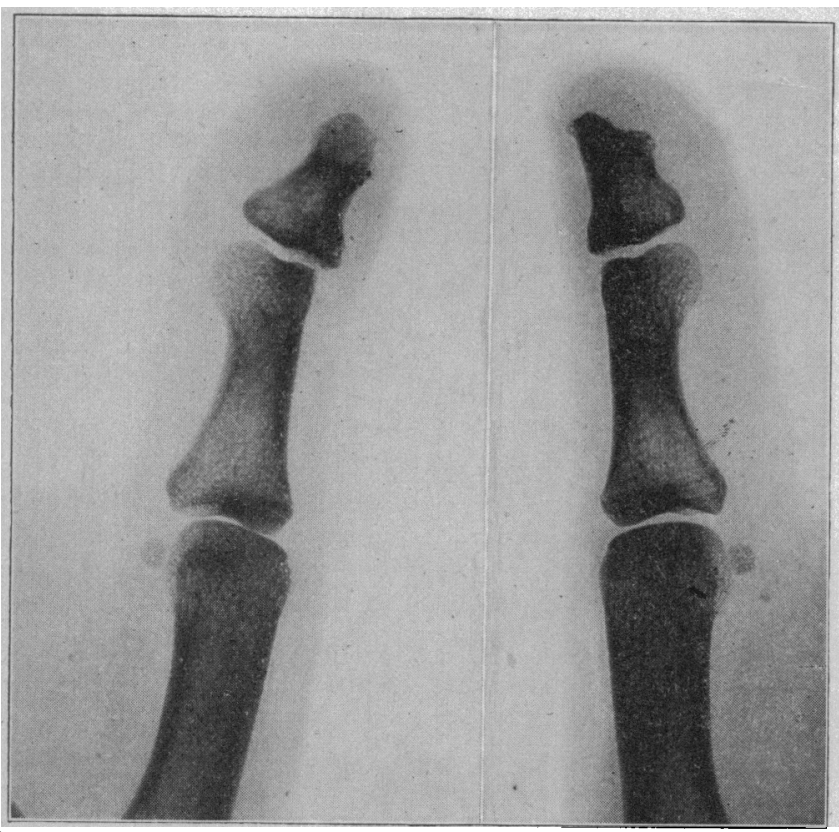

Fig. 2.-Regeneration of phalanges, January, 1917.

and dressed at intervals for this period. Amputation of the terminal phalanges was suggested, but was refused by the family, who at about this time moved to Newark and brought the child to me.

Examination revealed a sinus on the ball of each thumb, and loose bone could be detected on probing. A roentgeno- gram taken by Dr. C. F. Baker, Dec. 28, 1914, revealed complete destruction of the terminal phalanges of each thumb, extending to the epiphyseal line. There were loose sequestra, in each case, as shown in Figure 1. Under local anesthesia, the sinuses were enlarged and the sequestra removed. This was followed by healing in about three weeks.

No further attention was given the patient until January, 1917 , about two years later, when on examination bone had apparently reformed, and a second roentgenogram (Fig. 2) was taken, showing complete regeneration of the phalanges.

This case seems of particular interest, both from the standpoint of etiology (probably neurologic) and from the endresult.

\section{A CASE ILLUSTRATING FOREIGN BODIES IN THE STOMACH}

\section{E. L. Eliason, M.D., Philadelphia}

The patient was operated on in Dr. J. B. Carnett's service at the Philadelphia General Hospital. He was referred from

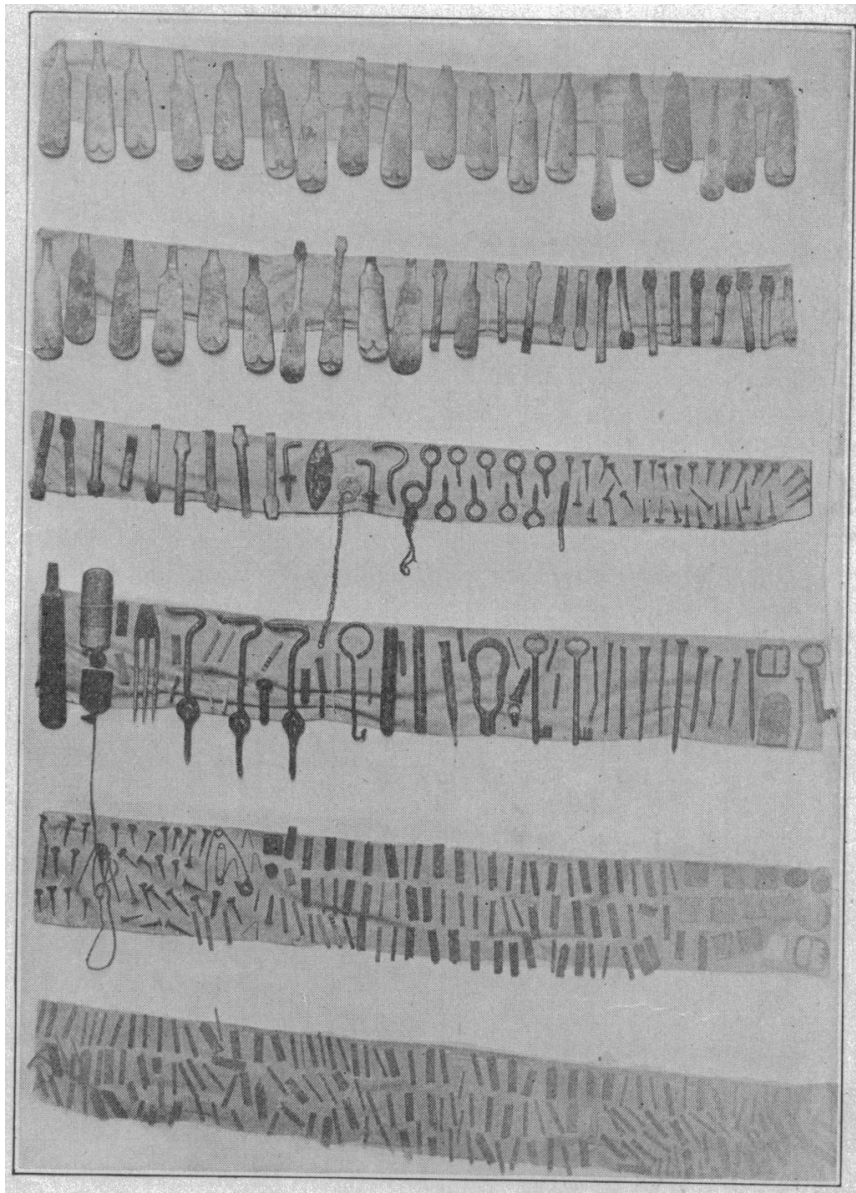

Foreign bodies removed from the stomach.

the men's nervous ward for operation for acute empyema of the gallbladder. At operation this condition was found, with 213 gallstones. In addition, 452 foreign bodies weighing 3 pounds were removed from the stomach. The patient made a good recovery.

REPORT OF CASE

J. S., man, admitted, June 16, 1917, to the neurologic service of Dr. Charles Potts in a rather pronounced state of melancholia, had a chill, July 4 , and his temperature rose to $105 \mathrm{~F}$. Physical examination revealed a rigid abciomen with marked tenderness and rigidity over the upper right rectus muscle. The leukocytic count was 11,800 .

When the patient was transferred to the surgical ward the following day, the temperature had fallen to 100, and the rigidity was less. A few days later, because of a return of the symptoms, a further examination of the abdomen was 
made. Rigidity and tenderness were again found over the gallbladder. In addition a mass was found with an irregular outline filling the right loin and extending across the abdomen and merging above with the liver. The mass on palpation was found to be movable, and gave the impression of a bag of stones. Dr. R. G. Torrey of the medical service reported the same findings, and further stated that the mass seemed distinct from the liver, but that it could not be clearl? outlined on account of muscle rigidity. A hypernephroma of the right kidney was thought of, as was also a retroperitoneal neoplasm. The patient had vomited a rusty brown fluid, which was examined for blood and found negative. No roentgenogram was taken.

July 16, I operated through a modified Martin J-shaped incision. The gallbladder was found enormously distended, greatly infiltrated, and covered with organizing lymph. The duodenum, colon and omentum were closely adherent to it. Filling the entire upper abdomen was a greatly hypertrophied stomach, filled with foreign bodies. The stomach was opened and found to be filled with 452 bodies consisting of nails, pins, tacks, spoons, hooks, glass, forks, etc., as shown in the illustration. The printers' type was wedged so tightly in the pyloric end of the stomach that it had to be picked apart by the finger before it would come loose. The stomach was emptied and closed by a triple tier suture. The gallbladder was next removed and found to be gangrenous and filled with pus and stones.

The patient was drained and returned to the ward. The postoperative course was rather stormy for forty-eight hours, but the patient then improved rapidly. On the third day the patient tried to remove the safety pin from his drainage tube and swallow it.

August 10, the bile drainage had stopped and the wound was almost entirely healed. Roentgenoscopy revealed no foreign bodies in the gastro-intestinal tract. The patient stated that he swallowed the first foreign body just three weeks before operation.

August 14, the patient was out of bed.

August 23 , the patient was surgically read" for discharge.

320 South Sixteenth Street.

THE USE OF CALCIUM CHLORID TO PRESERVE MOISTURE IN ANATOMIC SPECIMENS AND CADAVERS

William F. Hemler, M.D., Washington, D. C.

Assistant Professor of Anatomy, Georgetown University School of Medicine

On account of the high price of glycerin, we began to experiment, about a year ago in the laboratories of Georgetown University School of Medicine, to obtain if possible some other substance to keep anatomic specimens moist. As a result, we have adopted for this purpose purified calcium chlorid.

Brain specimens were first hardened in a 5 to 10 per cent. solution of formaldehyd, for about two months, and then transferred to the saturated solution of calcium chlorid, in which they remained for one week. They were then placed on ordinary dissecting room trays, without any cover, except another tray inverted to keep out the dust, but in no solution whatsoever and without even a damp cloth covering to retain moisture.

These specimens have now been kept in this manner for periods ranging from one to nine months, at ordinary room temperature and during the hot months of a Washington summer.

The specimens are perfectly moist and well preserved, and in these trays are readily accessible for study by the students. The gray and the white matter are to be distinguished better than in fresh brains.

By putting about 2 pounds in the embalming fluid for each cadaver, we have apparently been successful also in keeping them moist during dissection.

1330 East Capitol Street.

\section{Military Medicine and Surgery}

\author{
TENDON OPERATIONS FOR GUNSHOT \\ INJURIES OF THE HAND \\ LEO MAYER, A.M., M.D. \\ NEW YORK
}

In a recent series of articles ${ }^{1}$ I outlined the principles of a new system of tendon transplantations based on the anatomy and physiology of tendons. The essential principle of this system lies in the coordination of each step of the operation with the exact anatomic and physiologic facts, as ascertained by researches on the cadaver, by animal experimentation, and by operations on human beings. Thus, the tension under which the tendion is sutured conforms to the normal tension, a physiologic fact hitherto unknown; the transfer of the tendon from its original bed to the new site follows the laws governing the gliding

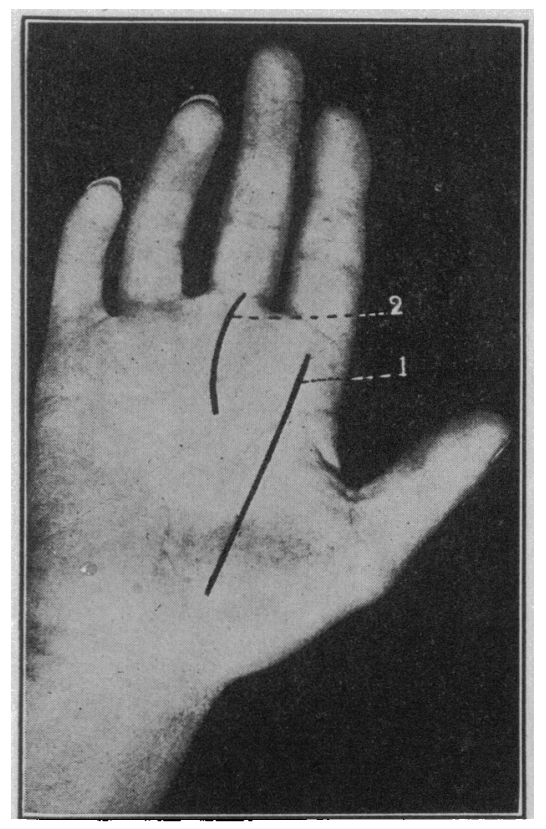

Fig. 1.-The incisions for transplantation of the flexor sublimis tendon of the index finger for the severed flexors of the middle finger: 1, first incision; 2, second incision. mechanism of tendons; the implantation of the tendons is such as to assure an anchorage as firm as the normal; even in the postoperative treatment the length of time allowed before exercise is begun is determined by exact experimental data. $T$ h e .results attained by the method, as observed in 250 cases of poliomyelitis and in fifty tendon transfers subsequent to gunshot injuries, justify its employment as a sound surgical procedure.

At the present time a double significance attaches to a rational system of tendon operations. First, there is the large group of poliomyelitis cases in which decided improvement of function can be obtained by tendon transplants; secondly, there will unquestionably, provided the war is not brought to an abrupt conclusion, be a large number of patients with gunshot injuries, on whom similar operations can be satisfactorily performed. That this group of patients will probably represent no small percentage of those to be treated in our American base hospitals is shown by my experience during a three years' service as surgeon to a 500 bed Red Cross base hospital near Berlin, where of 1,400 operations, fifty, or almost 4 per cent., were performed on tendons.

The purpose of this paper is to draw attention to several tendon transplantations hitherto not described, which I have found of service in gunshot injuries of the hand. One of the commonest lesions seen in a base

1. Mayer, Leo: Surg., Gynec. and Obst., 1916, 22, 182, 298 and 472. 